\title{
Drug-eluting bead trans-arterial chemoembolization combined with microwave ablation therapy vs. microwave ablation alone for early stage hepatocellular carcinoma: a preliminary investigation of clinical value
}

\author{
Juanfang Liu ${ }^{1} \cdot$ Wenguang Zhang ${ }^{1} \cdot$ Huibin $\mathrm{Lu}^{1} \cdot$ Hongbin $\mathrm{Li}^{1} \cdot$ Xueliang Zhou ${ }^{1} \cdot$ Jing Li $^{1} \cdot$ Xinwei Han $^{1}$ (1)
}

Received: 23 March 2021 / Accepted: 11 August 2021 / Published online: 17 August 2021

(c) The Author(s) 2021

\begin{abstract}
Purpose To assess the clinical value of drug-eluting bead trans-arterial chemoembolization (DEB-TACE) combined with microwave ablation (MWA) vs. MWA treatment alone for early stage hepatocellular carcinoma (HCC).

Materials and methods Consecutive data from $102 \mathrm{HCC}$ patients at early stage who were referred to our hospital from December 2014 to May 2016 were retrospectively collected. Forty-seven patients underwent DEB-TACE combined with MWA treatment, whereas 55 patients underwent MWA alone. After 1 month of treatment, the tumour responses of the patients were assessed using the mRECIST criteria. Treatment-related complications and hepatic function were also analysed for the two groups. In addition, overall survival (OS) and progression-free survival (PFS) were calculated and compared.

Results Patients in the combined treatment group (DEB-TACE combined with MWA) presented a better objective response rate (ORR) and disease control rate (DCR) compared with those in the monotherapy group (MWA treatment). The median OS and PFS were longer in the combined treatment group compared with the monotherapy group. Multivariate Cox's regression further illustrated that DEB-TACE + MWA vs. MWA was an independent protective factor for PFS and OS. No serious treatment-related complications were observed in any of the patients.

Conclusion Combined treatment with DEB-TACE appeared to have advantages in prolonging OS and PFS compared to MWA. Therefore, combined treatment was efficient and should be strongly recommended to early stage HCC patients.
\end{abstract}

Keywords Early stage hepatocellular carcinoma $\cdot$ Drug-eluting bead trans-arterial chemoembolization $\cdot$ Microwave ablation $\cdot$ Arsenic trioxide

\section{Introduction}

Hepatocellular carcinoma (HCC) is the third most common cause of cancer-related mortality worldwide (Bray et al. 2018). Consistent with the latest clinical practice guidelines

Xinwei Han

1021056466@qq.com

Juanfang Liu

wangxiaoou2015@126.com

Wenguang Zhang

zwg6499@163.com

Huibin Lu

lhb826@163.com

Hongbin Li

Lihongbin2012@foxmail.com for $\mathrm{HCC}$ issued by the European Association for the Study of the Liver (EASL), for early stage HCC patients who are defined as a single tumour $\leq 5 \mathrm{~cm}$ or three nodules $\leq 3 \mathrm{~cm}$ (Llovet et al. 2003), liver transplantation, surgical resection, and ablation are the recommended treatments (Galle et al.

Xueliang Zhou

15093286153@163.com

Jing Li

lijingerzzu@foxmail.com

Department of Interventional Radiology, The First Affiliated Hospital of Zhengzhou University, No. 1, East Jian She Road, Zhengzhou 450052, Henan, People's Republic of China 
2018). Recently, a minimally invasive and effective therapy has been highly praised by HCC patients. As the first choice for nonsurgical treatment, ablation has become a significant alternative treatment for small or early stage HCC. The efficiency of MWA has been confirmed repeatedly in the treatment of patients with tumours $\leq 5 \mathrm{~cm}$ (Kamal et al. 2019).

At present, arsenic trioxide (ATO) has been suggested to be an effective chemotherapeutic agent used primarily for solid tumours, such as HCC, colorectal, lung, bladder, breast, and pancreatic cancer (Huang et al. 2019; Kong et al. 2020; Kritharis et al. 2013; Wang et al. 2018). Transarterial chemoembolization (TACE) or DEB-TACE loading with ATO has been widely used and is effective in the treatment of human primary HCC. Given the advantage of avoiding systemic toxicity, drug-eluting bead trans-arterial chemoembolization (DEB-TACE) has been developed and widely used in combination therapy for HCC.

Combination therapy of TACE combined with thermal ablation yielded better treatment efficacy than monotherapy of MWA or TACE treatment in HCC patients, whereas DEBTACE combined with MWA for early stage HCC patients has not yet been investigated. Thus, we hypothesized that DEB-TACE and MWA combination therapy might offer promising outcomes for early stage HCC patients.

\section{Materials and methods}

\section{Patients}

This retrospective study was approved by the institutional review board and was conducted in accordance with the Declaration of Helsinki. Written informed consent was waived, because the study was retrospective. From December 2014 to May 2016, a total of 383 HCC patients who underwent DEB-TACE plus MWA or MWA treatment were screened. 102 patients with small HCC were enrolled in our study. Forty-seven patients underwent DEB-TACE combined with MWA treatment, whereas 55 patients underwent MWA alone. The main inclusion criteria were (1) early stage HCC; (2) Child Pugh A; (3) Eastern Cooperative Oncology Group performance status (ECOG PS 0 or 1); and (4) completed data. The screening flow chart of inclusion and exclusion criteria were exhibited in Fig. 1.

\section{Treatment schedule}

\section{DEB-TACE treatment}

Before DEB-TACE, the CalliSpheres Beads (Jiangsu Hengrui Medicine Co. Ltd., Jiangsu, China) with diameters of 300-500 $\mu \mathrm{m}$ were used as the carrier for loading with ATO (40-60 mg) (Beijing Shuanglu Pharmaceuticals,
Inc., China). During the loading procedure, the mixture was shaken every $5 \mathrm{~min}$ at room temperature to enable the beads to fully load with ATO. Once loaded, iodine alcohol was added at a ratio of 1:1 into the above mixture for further use. The DEB-TACE procedure was performed as described by Wu et al. (2018) and Liu et al. (2019). The patients in the experimental group underwent DEB-TACE at least once, and treatment times should take into account tumour recurrence.

\section{MWA treatment}

MWA was generally performed by the same team of doctors 1 week after the DEB-TACE procedure. The patients were under conscious sedation and local anaesthesia. After anaesthesia was attained, the MWA probe (ECO-100AI10, ECO Microwave System Co, Nanjing, China) was inserted into the lesion under the guidance of CT with an output power of 50-60 W for 5-10 min. The ablation time should consider the depth, size and surrounding structure of the target lesions. Coagulation of the needle tract was performed to prevent bleeding or tumour seeding after ablation. Finally, a CT scan was performed after ablation to verify the extent of the ablation region as well as whether abnormal bleeding occurred.

\section{Assessment of response and follow-up protocol}

The primary endpoints included overall survival (OS) and progression-free survival (PFS). The time of OS was the period from the first interventional treatment to death or last follow-up. PFS was deemed the duration from the time of first interventional treatment to the time of disease progression or death or last follow-up. The secondary endpoint was tumour response, including the objective response rate (ORR) and disease control rate (DCR) as described in the modified Response Evaluation Criteria in Solid Tumours (mRECIST). ORR was defined as the percentage of patients who achieved CR or PR, and DCR was defined as the proportion of patients who achieved CR, PR or SD. Complications were observed clinically during admission and assessed by telephone interview after discharge. Complications were graded based on the Common Terminology Criteria for Adverse Events (CTCAE, version 3.0). Laboratory tests and abdominal ultrasound or enhanced CT/MRI were reviewed to assess tumour response at 7 th day, first month, second month, third month and sixth month after the first treatment. Then, patients were followed up every 6 months. DEB-TACE + MWA or MWA was completed once more if tumour recurrence occurred. The last follow-up time was May, 15, 2021. 


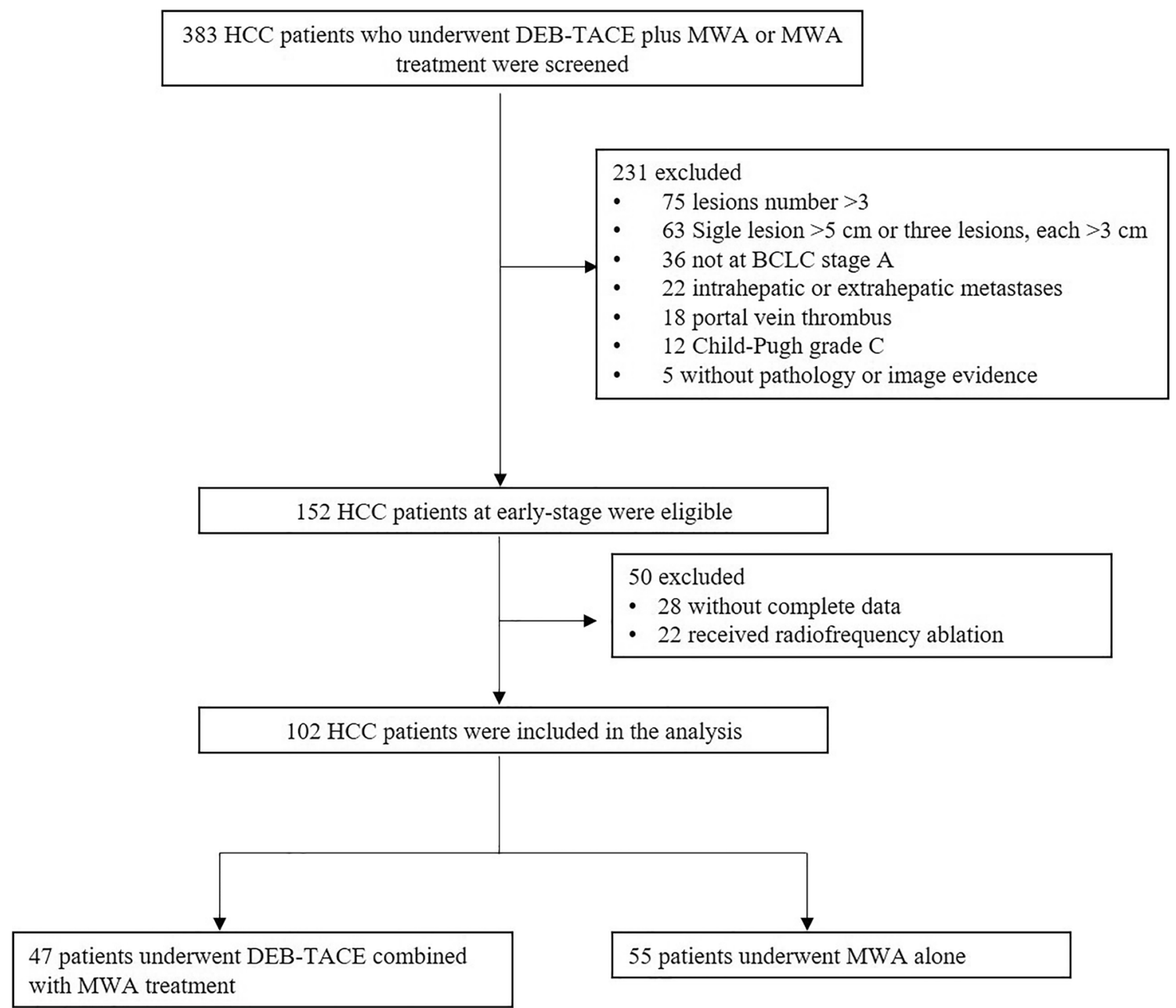

Fig. 1 Study flow. $H C C$ hepatocellular carcinoma, $D E B$-TACE drug-eluting bead trans-arterial chemoembolization, $M W A$ microwave ablation

\section{Statistical analysis}

Statistical analysis was performed using SPSS 22.0 statistical software (SPSS Inc., USA), and figures were created using GraphPad Prism 6.01 software (GraphPad Software Inc., USA). Categorical variables were expressed as numbers or percentages (\%), and continuous variables were expressed as the mean \pm standard deviation (SD) or median (25th-75th percentiles) as appropriate. Comparisons between two groups were determined by the Chisquare test, $t$ test or Wilcoxon rank sum test. Survival analysis was performed using the Kaplan-Meier method, and differences in survival profiles between two groups were determined by the log-rank test. Multivariate Cox's regression analyses were used to predict prognostic factors of PFS and OS. $P$ value $<0.05$ was considered significant.

\section{Results}

\section{Patient characteristics}

A total of $102 \mathrm{HCC}$ patients at early stage were included in the present study. There were no significant differences in age, sex, Child-Pugh classification, cause of cirrhosis, tumour size, AFP level or tumour location between the two groups. The average tumour size was not different between the two groups, with $3.49 \pm 0.88 \mathrm{~cm}$ in the DEBTACE + MWA group and $3.48 \pm 0.86 \mathrm{~cm}$ in the control (MWA only) group. The ratios of patients with 1, 2-3 nodules were $63.8 \%, 36.2 \%$ in the DEB-TACE + MWA group vs. $69.1 \%, 30.9 \%$ in the MWA alone group, and no significant differences were observed between the two groups. 


\section{Tumour response}

Tumour response was assessed on the basis of follow-up CT/MRI at the first month (M1) and sixth month (M6) after treatment. In the first month after treatment, the objective response rate (ORR) and disease control rate (DCR) were both slightly higher in the DEB-TACE + MWA group compared to the MWA alone group ( $100 \%$ vs. $94.5 \%, P=0.106$ and $100 \%$ vs. $98.2 \%, P=0.355$, respectively), and no significant differences were observed. However, at M6 after treatment, a significant difference in DCR $(93.6 \%$ vs. $78.2 \%$, $P=0.028$ ) was noted between the two groups. Similarly, a more promising ORR $(97.91 \%)$ was observed in the DEBTACE + MWA group, which was significantly different from that $(87.3 \%)$ in the control group $(P<0.05)$ (Table 1$)$.

\section{Survival}

The Kaplan-Meier method was used to assess the OS and PFS of HCC patients at early stage, and the difference between the DEB-TACE + MWA and MWA groups was determined using the log-rank test. At the end of the follow-up, a total of 50 patients died (16 patients in DEBTACE + MWA group, 34 in MWA group). 32 patients occurred progression including $22(51.1 \%)$ patients of intrahepatic recurrences and $8(17.0 \%)$ patients of extrahepatic metastasis in DEB-TACE + MWA group. In the MWA group, there were 44 patients progressed including $33(60.0 \%)$ of intrahepatic recurrences and 11 (20.0\%) patients of extrahepatic metastasis. The mean OS was significantly improved in the DEB-TACE + MWA group compared to the MWA group (55.67 months; 95\% CI 52.92-58.38 vs. 49.13 months; $95 \%$ CI $45.45-52.81$; $P=0.003$ ). The median OS was 60.0 months (inter quartile range, IQR 58.0-60.0 months) in the TACE + MWA group and 55.0 months (IQR, 37.0-60.0 months) in the MWA group. Similarly, the mean PFS was also significantly prolonged in the DEB-TACE + MWA group compared to the MWA alone group (47.61 months; 95\% CI 43.89-51.32 vs. 38.56 months; 95\% CI 34.26-42.86; $P=0.018$ ). Based on the Kaplan-Meier curve, the cumulative survival rates at 1, 3 and 5 years were $100 \%, 89.4 \%$ and $65.7 \%$, respectively, in the DEB-TACE + MWA group and $98.2 \%, 80.0 \%$ and $36.1 \%$, respectively, in the MWA group (Fig. 2).

\section{Factors affecting OS and PFS}

Univariable Cox proportional hazard regression analysis indicated that Child-Pugh class (B vs. A) (both $P<0.001$ ) and numbers of lesions (2-3 vs. 1$)$ (both $P<0.005$ ) were associated with shorter OS and PFS. However, DEBTACE + MWA vs. MWA showed a longer OS and PFS $(P=0.005$ and $P=0.021$, respectively) (Table 2). Multivariable Cox regression revealed that numbers of lesions (2-3 vs. 1) (both $P<0.001$ ) independently predicted worse OS and PFS in HCC patients at early stage. While, DEBTACE + MWA vs. MWA predicted longer OS and PFS (both $P<0.001$ ) (Table 3).

\section{Adverse reactions and complications}

The most common adverse events in patients who underwent DEB-TACE were gastrointestinal reaction, liver dysfunction, and bone-marrow suppression. Typical complications related to MWA included pain and fever. There was no significant difference in the incidence of complications related to MWA between the two groups $(P>0.05$, all). Of the 47 patients who underwent DEB-TACE + MWA treatment, 12 patients had bone-marrow suppression, and 9 patients had nausea and vomiting. All adverse events and complications were classified as grade 1-2 according to Common Terminology Criteria for Adverse Event (CTCAE 3.0), including 9 patients who experienced pleural effusion (4 in the DEB-TACE + MWA group, 5 in the MWA group, $P>0.05$ ), 8 patients who developed new ascites ( 3 in the TACE + MWA group, 5 in the MWA group, $P>0.05)$, and 10 patients who developed subcapsular liver haemorrhage (4 in the TACE + MWA group, 6 in the MWA group, $P>0.05$ ). All patients were corrected after symptomatic treatment. No serious treatment-related deaths were observed (Table 4).
Table 1 Treatment response between the two groups

\begin{tabular}{lllllllll}
\hline Response & CR & PR & SD & PD & ORR & P1 & DCR & P2 \\
\hline M1 & & & & & & & & \\
$\quad$ Experiment group $(n=47)$ & 45 & 2 & 0 & 0 & 100 & 0.106 & 100 & 0.355 \\
$\quad$ Control group $(n=55)$ & 50 & 2 & 2 & 1 & 94.5 & & 98.2 & \\
M6 & & & & & & & & \\
Experiment group $(n=47)$ & 42 & 2 & 2 & 1 & 93.6 & $\mathbf{0 . 0 2 8}$ & 97.9 & $\mathbf{0 . 0 4 7}$ \\
$\quad$ Control group $(n=55)$ & 42 & 1 & 5 & 7 & 78.2 & & 87.3 & \\
\hline
\end{tabular}

$M 1$ first month after TACE therapy, $M 6$ sixth month after TACE therapy, $C R$ complete response, $P R$ partial response, $S D$ stable disease; $\mathrm{PD}$ : progressive disease; objective response rate $(\mathrm{ORR}) \mathrm{CR}+\mathrm{PR}$, disease control rate $(D C R) \mathrm{CR}+\mathrm{PR}+\mathrm{SD}$ 


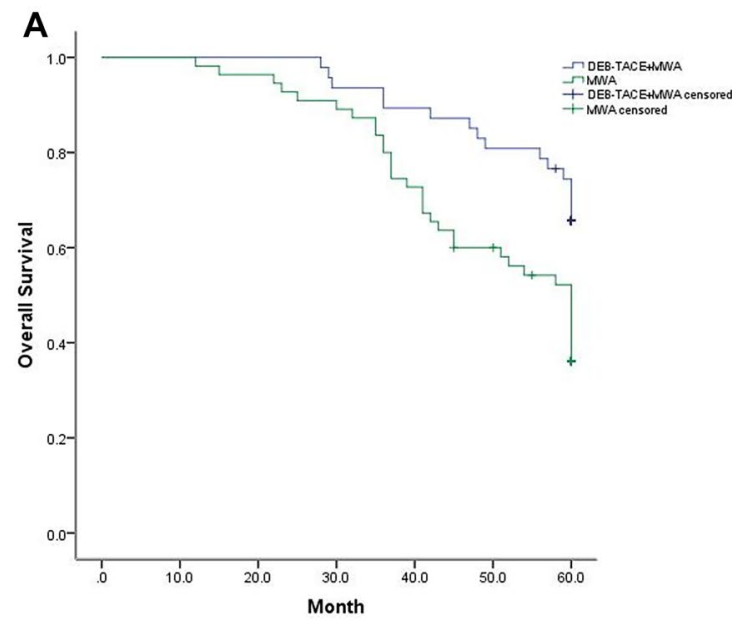

Fig. 2 OS and PFS between DEB-TACE + MWA and MWAE groups. Kaplan-Meier method was used to assess OS and PFS. A DEBTACE + MWA significantly prolonged the mean OS compared with MWA monotherapy. B Kaplan-Meier survival curve demonstrating longer mean PFS in the DEB-TACE+MWA group compared with

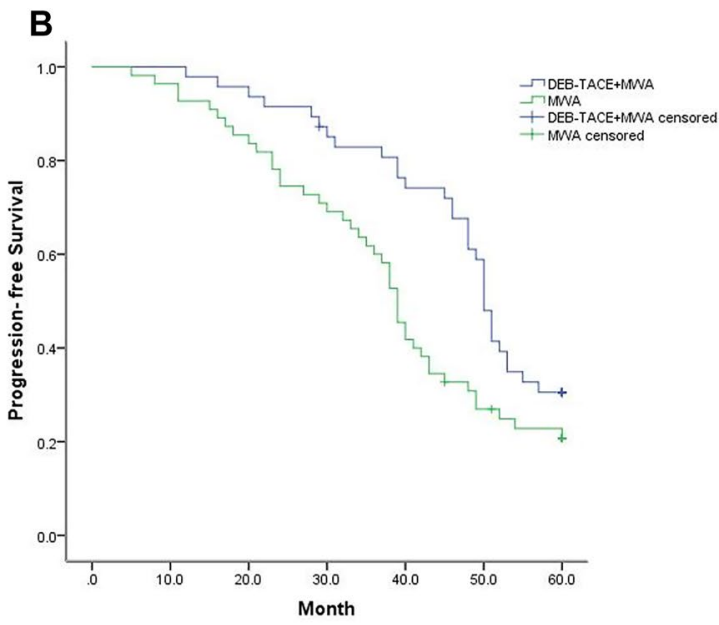

the MWA alone group. DEB-TACE, drug-eluting bead trans-arterial chemoembolization; TACE, trans-arterial chemoembolization; MWA, microwave ablation; OS, overall survival; PFS, progression-free survival
Table 2 Univariate Cox's proportional hazards regression model analysis for OS and PFS

\begin{tabular}{|c|c|c|c|c|c|c|c|c|}
\hline \multirow[t]{3}{*}{ Parameters } & \multicolumn{3}{|l|}{ OS } & \multirow{3}{*}{$P$} & \multicolumn{3}{|l|}{ PFS } & \multirow{3}{*}{$P$} \\
\hline & \multirow[t]{2}{*}{ HR } & \multicolumn{2}{|c|}{$95 \% \mathrm{CI}$} & & \multirow[t]{2}{*}{ HR } & \multicolumn{2}{|c|}{$95 \% \mathrm{CI}$} & \\
\hline & & Lower & Higher & & & Lower & Higher & \\
\hline \multicolumn{9}{|l|}{ Univariate Cox's regression } \\
\hline Age $(\geq 60$ vs. $<60)$ & 1.427 & 0.800 & 2.544 & 0.228 & 0.866 & 0.531 & 1.478 & 0.642 \\
\hline Gender (Male vs. Female) & 1.382 & 0.649 & 2.944 & 0.401 & 1.296 & 0.682 & 2.462 & 0.428 \\
\hline $\operatorname{AFP}(>400 \mathrm{vs} . \leq 400 \mathrm{ng} / \mathrm{mL})$ & 1.322 & 0.759 & 2.303 & 0.324 & 0.901 & 0.570 & 1.424 & 0.655 \\
\hline Child-Pugh grade (B vs. A) & 4.835 & 2.725 & 8.580 & $<0.001$ & 3.339 & 2.046 & 5.448 & $<0.001$ \\
\hline Number (2-3 lesions vs. 1) & 5.431 & 3.061 & 9.637 & $<0.001$ & 4.844 & 3.013 & 7.790 & $<0.001$ \\
\hline Location (left vs. right) & 0.970 & 0.455 & 2.066 & 0.937 & 1.130 & 0.621 & 2.055 & 0.690 \\
\hline DEB-TACE + MWA vs. MWA & 0.423 & 0.233 & 0.767 & 0.005 & 0.581 & 0.366 & 0.921 & 0.021 \\
\hline
\end{tabular}

$O S$ overall survival, $H R$ hazard ratio, $C I$ confidence interval, $P F S$ progression free survival

Table 3 Multivariate Cox's proportional hazards regression model analysis for OS and PFS

\begin{tabular}{|c|c|c|c|c|c|c|c|c|c|c|c|c|}
\hline \multirow[t]{3}{*}{ Parameters } & \multicolumn{6}{|l|}{ OS } & \multicolumn{6}{|l|}{ PFS } \\
\hline & \multirow[t]{2}{*}{$B$} & \multirow[t]{2}{*}{ Wald } & \multirow[t]{2}{*}{ HR } & \multicolumn{2}{|l|}{$95 \% \mathrm{CI}$} & \multirow[t]{2}{*}{$P$} & \multirow[t]{2}{*}{$B$} & \multirow[t]{2}{*}{ Wald } & \multirow[t]{2}{*}{ HR } & \multicolumn{2}{|l|}{$95 \% \mathrm{CI}$} & \multirow[t]{2}{*}{$P$} \\
\hline & & & & Lower & Higher & & & & & Lower & Higher & \\
\hline Child-Pugh grade (B vs. A) & 0.083 & 0.011 & 1.086 & 0.342 & 3.447 & 0.888 & 0.061 & 0.025 & 1.062 & 0.442 & 2.549 & 0.894 \\
\hline DEB-TACE + MWA vs. MWA & -1.829 & 17.392 & 0.160 & 0.077 & 0.332 & $<0.001$ & -1.189 & 18.497 & 0.304 & 0.178 & 0.517 & $<0.001$ \\
\hline Number (2-3 lesions vs. 1 ) & 1.898 & 19.415 & 6.667 & 3.052 & 14.564 & $<0.001$ & 1.765 & 27.283 & 5.840 & 3.057 & 11.158 & $<0.001$ \\
\hline
\end{tabular}

\section{Discussion}

TACE can selectively deliver chemotherapeutics to targeted lesions, leading to tissue necrosis by reducing the local blood supply of tumour lesions. However, the tumour recurrence rate was relatively high after TACE. Recently, many studies have revealed that patients with HCC treated with DEB-TACE tend to present a better response and tolerance than those who undergo TACE treatment (Liu et al. 
Table 4 Adverse reactions and complications

\begin{tabular}{lrlr}
\hline & $\begin{array}{l}\text { DEB- } \\
\text { TACE+MWA } \\
(n=47)\end{array}$ & MWA (n=55) & $P$ \\
& $19(40.4)$ & $16(29.1)$ & 0.229 \\
Abdominal pain & $15(31.9)$ & $16(29.1)$ & 0.757 \\
Fever & $14(29.8)$ & $11(20.0)$ & 0.252 \\
Inappetence & $4(8.5)$ & $5(9.1)$ & 0.918 \\
Pleural effusion & $4(8.5)$ & $6(10.9)$ & 0.685 \\
Subcapsular liver haem- & & & \\
$\quad$ orrhage & $3(6.4)$ & $3(5.5)$ & 0.843 \\
Liver abscess & $3(6.4)$ & $5(9.1)$ & 0.612 \\
New ascites & $13(27.7)$ & $11(20.0)$ & 0.363 \\
Liver dysfunction & $7(14.9)$ & $6(10.9)$ & 0.548 \\
Hypoalbuminemia & $8(17.0)$ & $5(9.1)$ & 0.231 \\
Bilirubin elevation & $9(19.1)$ & $1(1.8)$ & $\mathbf{0 . 0 0 3}$ \\
Nausea and vomiting & $12(25.5)$ & 0 & $<\mathbf{0 . 0 0 1}$ \\
Bone-marrow suppres- & & & \\
$\quad$ sion & & & \\
\hline
\end{tabular}

2019; Woo and Heo 2015; Wu et al. 2018; Xiao et al. 2019; Zhou et al. 2018). DEB-TACE has the ability to control the speed of antitumour drug release, which can provide prolonged and sustained drug delivery and a high diffusion of chemotherapeutics into the local target lesion, resulting in a mild systemic reaction due to a lower concentration of chemotherapeutics in serum. As a chemotherapeutic agent, ATO is approved as a chemotherapy for HCC and other solid tumours in our country. Wang $\mathrm{H}$ demonstrated that locoregional therapy combined with ATO treatment could prolong the survival time of HCC patients (Wang et al. 2015). Similarly, our team previously demonstrated that DEB-TACE with ATO is more effective than TACE in patients with unresectable HCC (Duan et al. 2020).

Synergistic effect of TACE and MWA is ideal. Currently, combination therapy of TACE and MWA is effective and widely used in clinical practice ( $\mathrm{Li}$ et al. 2020; Wang et al. 2019). In this study, we first assessed the efficacy and safety of DEB-TACE + MWA compared with MWA alone in early stage HCC patients. A study performed by Shi F et al. showed an ideal result: the OS rates were $100 \%, 79 \%$, and $73 \%$ at 1,3 , and 5 years, respectively, in patients with HCC outside the Milan group who underwent TACE + MWA treatment (Shi et al. 2020). A retrospective study conducted by Zheng $\mathrm{L}$ showed better OS rates in the TACE + MWA group compared with the TACE alone group in patients with large HCC, with $1-, 2$-, and 3-year OS rates of $85.9 \%, 59.8 \%$, and $32.6 \%$ vs. $59.0 \%, 40.4 \%$, and $11.4 \%$, respectively (Zheng et al. 2018). Wei J and colleagues confirmed that TACE + MWA had superiority in prolonging median OS and reducing TTP compared with TACE + cryoablation (CRA) in treating unresectable HCC (Wei et al. 2020). In addition, our results showed that the OS rates at 1,3 , and 5 years in DCB-TACE + MWA group were $100 \%, 89.4 \%$ and $65.7 \%$, respectively, which were similar with those reported by $\mathrm{Li} \mathrm{Z}$; the OS rates at 1, 3 and 5 years were $98.0 \%, 89.8 \%$, and $74.3 \%$, respectively, in patients with small HCCs treated with TACE + MWA (Li et al. 2020). Patients in the DEB-TACE + MWA treatment group achieved a prolonged mean OS (55.66 months) and an improved median PFS (47.61 months). In contrast, shorter median OS and PFS (49.13 months and 38.56 months, respectively) were obtained in the MWA alone group. At M6, more promising ORR (93.6\%) and DCR (97.9\%) were observed in the DEB-TACE + MWA group compared with the MWA group (78.2\% and $87.3 \%$, respectively). Recently, a meta-analysis suggested that TACE + MWA was efficient and safe for unresectable HCC patients with prolonged OS and tolerable adverse reactions (Wang et al. 2019). In this study, multivariable Cox regression analysis revealed that there was a significant correlation of shorter median OS and PFS with tumour numbers of 2-3, while DEB-TACE + MWA independently predicted a longer OS and PFS.

The results of this study are encouraging. TACE is the preferred treatment for HCC patients in all stages with well-tolerated liver function and is performed prevalently worldwide. However, TACE alone could not be sufficient for all HCC patients. Recently, MWA exhibits superiority in treating small HCC lesions ( $\mathrm{Li}$ et al. 2021); nevertheless, this method is also affected by the cooling effect (Xu et al. 2019). The reasons are summarized as follows: (1) Tumour embolization caused by TACE could reduce the "cooling effect" of tumoral arterial blood flow. (2) The reduced or stabilized size of tumour after TACE would facilitate MWA treatment. (3) Ischemia and inflammatory edema, of tumour tissues occurred after TACE procedure, which had effect on increasing thermal effects of MWA. (4) MWA might destroy some hypovascular HCCs that were refractory to TACE procedures (Zheng et al. 2018). In this study, we substituted DEB-TACE for TACE given its better response and tolerance. Therefore, we administered DEB-TACE and MWA combination therapy, which can overcome these weaknesses and improve the efficacy of monotherapy.

Our study had several limitations. On the one hand, this was a retrospective study with a relatively small sample size, and the statistical power of our results might be mitigated. On the other hand, the breathing movement of patients might affect the accuracy of punctures under CT guidance. Therefore, further randomized controlled trials or prospective studies with larger sample sizes are needed to verify the results.

In conclusion, combination therapy of DEB-TACE with MWA prolonged the survival time of early stage HCC patients in comparison with MWA alone. Hence, treatment of DEB-TACE with ATO+MWA is safe and efficient, which 
may offer a promising new approach for the management of early stage HCC.

\begin{abstract}
Author contributions All authors contributed to the study conception and design. Material preparation, data collection and analysis were performed by WZ, HL, XZ, JL and HL. The first draft of the manuscript was written by $\mathrm{JL}$ and $\mathrm{XH}$ and all authors commented on previous versions of the manuscript. All authors read and approved the final manuscript.
\end{abstract}

Funding There is no funding related to this study.

Availability of data and materials The data used to support the findings of this study are available from the corresponding author upon request.

Code availability Not Applicable.

\section{Declarations}

Conflict of interest The authors declare no conflicts of interest.

Ethical approval All procedures performed in studies involving human participants were in accordance with the ethical standards of the institutional and national research committee (The First Affiliated Hospital of Zhengzhou University, NO. yfyky2019-6231) and with the 1964 Helsinki declaration and its later amendments or comparable ethical standards.

Consent to participate Verbal informed consent was obtained prior to the interview.

Open Access This article is licensed under a Creative Commons Attribution 4.0 International License, which permits use, sharing, adaptation, distribution and reproduction in any medium or format, as long as you give appropriate credit to the original author(s) and the source, provide a link to the Creative Commons licence, and indicate if changes were made. The images or other third party material in this article are included in the article's Creative Commons licence, unless indicated otherwise in a credit line to the material. If material is not included in the article's Creative Commons licence and your intended use is not permitted by statutory regulation or exceeds the permitted use, you will need to obtain permission directly from the copyright holder. To view a copy of this licence, visit http://creativecommons.org/licenses/by/4.0/.

\section{References}

Bray F, Ferlay J, Soerjomataram I, Siegel RL, Torre LA, Jemal A (2018) Global cancer statistics 2018: GLOBOCAN estimates of incidence and mortality worldwide for 36 cancers in 185 countries. CA Cancer J Clin 68(6):394-424. https://doi.org/10.3322/ caac. 21492

Duan XH, Ju S, Han XW, Ren JZ, Li FY, Chen PF, Wu YY, Li H (2020) Arsenic trioxide-eluting Callispheres beads is more effective and equally tolerant compared with arsenic trioxide/lipiodol emulsion in the transcatheter arterial chemoembolization treatment for unresectable hepatocellular carcinoma patients. Eur Rev Med Pharmacol Sci 24(3):1468-1480

Galle PR, Forner A, Llovet JM, Mazzaferro V, Piscaglia F, Raoul J-L, Vilgrain V (2018) EASL clinical practice guidelines: management of hepatocellular carcinoma. J Hepatol. https://doi.org/10.1016/j. jhep.2018.03.019

Huang Y, Zhou B, Luo H, Mao J, Huang Y, Zhang K, Shan H (2019) ZnAs@SiO2 nanoparticles as a potential anti-tumor drug for targeting stemness and epithelial-mesenchymal transition in hepatocellular carcinoma via SHP-1/JAK2/STAT3 signaling. Theranostics 9(15):4391-4408. https://doi.org/10.7150/thno.32462

Kamal A, Elmoety AAA, Rostom YAM, Shater MS, Lashen SA (2019) Percutaneous radiofrequency versus microwave ablation for management of hepatocellular carcinoma: a randomized controlled trial. J Gastrointest Oncol 10(3):562-571. https://doi.org/10. 21037/jgo.2019.01.34

Kong D, Jiang T, Liu J, Jiang X, Liu B, Lou C, Feng G (2020) Chemoembolizing hepatocellular carcinoma with microsphere cored with arsenic trioxide microcrystal. Drug Deliv 27(1):1729-1740. https://doi.org/10.1080/10717544.2020.1856219

Kritharis A, Bradley TP, Budman DR (2013) The evolving use of arsenic in pharmacotherapy of malignant disease. Ann Hematol 92(6):719-730. https://doi.org/10.1007/s00277-013-1707-3

Li Z, Hao D, Jiao D, Zhang W, Han X (2020) Transcatheter arterial chemoembolization combined with simultaneous cone-beam computed tomography-guided microwave ablation in the treatment of small hepatocellular carcinoma: clinical experiences from 50 procedures. Acad Radiol. https://doi.org/10.1016/j.acra.2020.08.036

Li Z, Jiao D, Si G, Han X, Zhang W, Li Y, Zhou X, Liu J, Li J, Liu Z (2021) Making timely remedial measures after TACE based on the results of cone-beam CT liver perfusion. Int J Hyperthermia 38(1):428-436

Liu J, Xu J, Zhang W, Chen J, Zhou X, Li Z, Han X (2019) Safety and efficacy of drug-eluting bead transarterial chemoembolization combined with apatinib in patients with advanced hepatocellular carcinoma. Acad Radiol 27(5):704-709. https://doi.org/10.1016/j. acra.2019.07.003

Llovet JM, Burroughs A, Bruix J (2003) Hepatocellular carcinoma. The Lancet 362(9399):1907-1917. https://doi.org/10.1016/s01406736(03)14964-1

Shi F, Lian S, Mai Q, Mo Z, Zhuang W, Cui W, Chen X (2020) Microwave ablation after downstaging of hepatocellular carcinoma: outcome was similar to tumor within Milan criteria. Eur Radiol 30(5):2454-2462. https://doi.org/10.1007/s00330-019-06604-y

Wang H, Liu Y, Wang X, Liu D, Sun Z, Wang C, Yu S (2015) Randomized clinical control study of locoregional therapy combined with arsenic trioxide for the treatment of hepatocellular carcinoma. Cancer 121(17):2917-2925. https://doi.org/10.1002/cncr. 29456

Wang L, Min Z, Wang X, Hu M, Song D, Ren Z, Wang Y (2018) Arsenic trioxide and sorafenib combination therapy for human hepatocellular carcinoma functions via up-regulation of TNF-related apoptosis-inducing ligand. Oncol Lett 16(3):3341-3350. https:// doi.org/10.3892/ol.2018.8981

Wang L, Ke Q, Lin N, Huang Q, Zeng Y, Liu J (2019) The efficacy of transarterial chemoembolization combined with microwave ablation for unresectable hepatocellular carcinoma: a systematic review and meta-analysis. Int J Hyperthermia 36(1):1288-1296. https://doi.org/10.1080/02656736.2019.1692148

Wei J, Cui W, Fan W, Wang Y, Li J (2020) Unresectable hepatocellular carcinoma: transcatheter arterial chemoembolization combined with microwave ablation vs. combined with cryoablation. Front Oncol 10:1285. https://doi.org/10.3389/fonc.2020.01285

Woo H, Heo J (2015) Transarterial chemoembolization using drug eluting beads for the treatment of hepatocellular carcinoma: now and future. Clin Mol Hepatol 21(4):344-348

Wu B, Zhou J, Ling G, Zhu D, Long Q (2018) CalliSpheres drugeluting beads versus lipiodol transarterial chemoembolization in the treatment of hepatocellular carcinoma: a short-term efficacy 
and safety study. World J Surg Oncol 16(1):69. https://doi.org/10. 1186/s12957-018-1368-8

Xiao YD, Ma C, Zhang ZS, Liu J (2019) Safety and efficacy assessment of transarterial chemoembolization using drug-eluting beads in patients with hepatocellular carcinoma and arterioportal shunt: a single-center experience. Cancer Manag Res 11:1551-1557. https://doi.org/10.2147/CMAR.S193948

Xu Z, Xie H, Zhou L, Chen X, Zheng S (2019) The Combination strategy of transarterial chemoembolization and radiofrequency ablation or microwave ablation against hepatocellular carcinoma. Anal Cell Pathol 2019:1-7. https://doi.org/10.1155/2019/8619096

Zheng L, Li HL, Guo CY, Luo SX (2018) Comparison of the efficacy and prognostic factors of transarterial chemoembolization plus microwave ablation versus transarterial chemoembolization alone in patients with a large solitary or multinodular hepatocellular carcinomas. Korean J Radiol 19(2):237-246. https://doi.org/10. 3348/kjr.2018.19.2.237

Zhou GH, Han J, Sun JH, Zhang YL, Zhou TY, Nie CH, Zheng SS (2018) Efficacy and safety profile of drug-eluting beads transarterial chemoembolization by CalliSpheres(R) beads in Chinese hepatocellular carcinoma patients. BMC Cancer 18(1):644. https://doi.org/10.1186/s12885-018-4566-4

Publisher's Note Springer Nature remains neutral with regard to jurisdictional claims in published maps and institutional affiliations. 